\title{
Synthesis and Characterization of New 1,3-Oxazol-5-(4H)-one Derivatives
}

\author{
Abdul- Jabbar K. AL. Abodi", Ngam Majed., Sahar A. K. , Redha I. H. Al-Bayati \\ Department of chemistry, college of science AL- Mustansirya University, Baghdad, Iraq
}

\begin{abstract}
New 1,3-oxazol-5(4H)-one (3a- b) was synthesized by cyclization of[(Pyridyl-3-yl-carbonyl)amino]acetic acid (2). The starting were readily obtained by acylation of 2-a mino acetic acid (Glycine) with nicotinoyl chloride. Imidazo le was synthesized by reaction of compounds (3a-b) with hydrazine hydrate $(99 \%)$. Compounds $(4 a-b)$ were converted into a variety of derivatives. All new compounds were characterized by HNM and FTIR spectroscopy.
\end{abstract}

Keywords Nicotinic Acid, 1,3-Oxazole, Triazole, Thiadiazole, Thiourease

\section{Introduction}

The synthesis of 1,3-oxazoles have interest due to their various biological activities. Reported some of these activities were: nervous system depression[1,2], antibacteri al[3], herbicidal[4], and muscle relaxant[5] activities. Imidazole is a planer heterocyclic compound with five-member ring has three carbon atoms and two nitrogen atomat 1,3 positions. purine, $h$ is tamine, $h$ is tidine and nucle ic acid, including imidazole ring[6], Imidazole derivatives poss ess a broad spectrum of pharmacological activities such as Anti-fungal and Anti-bacterial activity[7]

Anticonvulsant[8] anti-Parkinson[9] and monoamine oxidase (MAO) inhibitory[10] activity. Products of Compounds containing a $1 H-1,2,4$ - triazole derivatives and many mono-, di- and tri-substituted 1,2,4-triazole-5-thiones Polyamide containing $N$-methylimidazole (Im) can be combined in antiparallel side-by-side dimericcomplexes with the minor groove of DNA[11], also compound 2-A mino-3-(4-carboxy-1H-imidazol-1-yl) propionic acid has been examined as an inhibitor of diaminopimelic acid dehydrogenase from Bacillus sphaericus [12]

\section{Experimental}

\subsection{General}

Melting point were determined in open capillary tubes on a Gallen kamp melting point apparatus and are uncorrected. The IR Spectra were recorded by $\mathrm{KBr}$ discs using a perkin-Elmer 1600 series FTIR spectrometer. 1HNMR

* Corresponding author:

chemabdu@yahoo.com (Abdul- Jabbar K. AL. Abodi)

Published online at http://journal.sapub.org/ajoc

Copyright (C) 2012 Scientific \& Academic Publishing. All Rights Reserved
Spectra were recorded on a Varian-Mercury 200 MHZ Spectrometer.

\subsection{Synthesis of Nicotinoyl Chloride (1)}

To a solution of nicotinic acid $(1.23 \mathrm{~g}, 0.01$ mole $)$ in dry benzene $(20 \mathrm{ml})$, thionyl chloride $(1.19 \mathrm{~g}, 0.01 \mathrm{~mol})$ was added. Then, the reaction mixture was refluxed for $7 \mathrm{~h}$. After evaporation, the product was collected without recrystallization. (yield $86 \%),\left(\mathrm{m} . \mathrm{p}, \mathrm{C}^{\circ}\right)(200-203)$, IR. $(\mathrm{KBr})$ (v, $\left.\mathrm{Cm}^{-1}\right) 3066$ (C-H Ar.), 1799 (C=O, acid chloride) 768 (C-Cl).

\subsection{Synthesis of [(Pyridyl-3-yl-Carbonyl Amino] Acetic Acid (2)}

To a stirring solution of glycine $(0.75 \mathrm{~g}, 0.01 \mathrm{~mol})$ and sodium hydroxide (10 ml,10\% solution), compound 1 (1.41 $\mathrm{g}, 0.01 \mathrm{~mol})$ was added. Then, the reaction mixture was shacked vigorously for $1 \mathrm{~h}$, , a few grams of crushed ice was added with stirring. After that, the solution was acidified with conc. $\mathrm{HCl}$ and the product was collected and recrystallized from ethanol. (yield $67 \%$ ), (m.p, $\left.\mathrm{C}^{\circ}\right)(250-253)$, ( IR. (KBr) $\left(v, \mathrm{Cm}^{-1}\right) 3180(\mathrm{NH}), 3178$ (acid $\mathrm{OH}$ ), 2976-2888 (C-H alph.) ,1741 (acid $\mathrm{C}=\mathrm{O}$ ), 1690 (amide $\mathrm{C}=\mathrm{O}) ;{ }^{1} \mathrm{HNMR}$ (DMSO-d6) $\varsigma$ (pp m) 3.31 (s, NH), 4.42 ( s, $\mathrm{CO}-\mathrm{CH} 2-\mathrm{NH}), 7.15-8.20$ ( $\mathrm{m}$, Aro matic protons).

\subsection{Synthesis of \\ 4-(Aryli dene)-2-(Pyri din-3-yl)-1,3-Oxazol-5(4H)- One(3a- B)}

To a stirring mixture of compound 2 (1.8 g, $0.01 \mathrm{~mol})$ acetic acid $(5 \mathrm{ml})$ acetic anhydride $(20 \mathrm{ml})$, aromatic aldehyde $(0.01 \mathrm{~mol})$ was added. The temperature of reaction was reached to $70 \mathrm{C}^{\circ}$ for $10 \mathrm{~min}$., then the mixture was poured in to crushed ice and stirred for $30 \mathrm{~min}$. the product was collected and recrystallized from ethanol to afforded the desired compound. 


\subsection{1. (3a). 4-(4-Bro mobenzylidene)-2-(Pyrid in-3-yl)-1, 3-Oxazo 1-5(4h)-One}

(yield $71 \%$ ), (m.p, $\left.\mathrm{C}^{\circ}, 120-123\right)$, IR. (KBr) $\left(v, \mathrm{Cm}^{-1}\right) 3050$ (C-H ar), 3123 (C-H olifen), 1755 (oxazole $\mathrm{C}=\mathrm{O}$ ), 1656 (oxazole $\mathrm{C}=\mathrm{N}$ ), $1600-1511(\mathrm{C}=\mathrm{C}$ Ar), $1280(\mathrm{C}-\mathrm{O}) 848$ (para substitution); ${ }^{1} \mathrm{HNMR}$ (DM SO-d6) $\varsigma$ (ppm) 8.90 ( s, $\mathrm{C}=\mathrm{CH}-$ ), $6.91-8.1$ (m, Aro matic protons)

\subsection{2. (3b ). 4-(4-Nitrobenzy lidene)-2-(Pyrid in-3-yl)-1, 3-Oxazo 1-5(4h)-One}

(yield $63 \%),\left(\mathrm{m} . \mathrm{p}, \mathrm{C}^{\circ}, 80-82\right), \mathrm{IR} .(\mathrm{KBr})\left(v, \mathrm{Cm}^{-1}\right) 3075$ (C-H ar), 3220 (C-H olifen), 1770 (oxazole $\mathrm{C}=\mathrm{O}$ ), 1650 (oxazole $\mathrm{C}=\mathrm{N}), 1600-1505(\mathrm{C}=\mathrm{C}$ Ar), $1230(\mathrm{C}-\mathrm{O}) 828$ (para substitution); ${ }^{1} \mathrm{HNMR}$ (DMSO-d6) $\varsigma$ (ppm) $8.82(\mathrm{~s}, \mathrm{C}=\mathrm{CH}-$ ), $7.21-8.1$ ( $\mathrm{m}$, Aro matic protons)

\subsection{Synthesis of 3-Amino-5-(Aryli dene)-2-(Pyridin-3-yl)- 3,5-Dihydro-4H-Imi dazol-4-One (4a-B)}

To a mixture of compound $(3 \mathrm{a}-\mathrm{b})(0.01 \mathrm{~mol})$ in dry pyridine $(5 \mathrm{ml})$ hydrazine hydrate $(99 \%)(10 \mathrm{ml})$ was added. The reaction mixture was refluxed for $20 \mathrm{~h}$. Then, the mixture was allowed to cool to room temperature and pyridine was removed. The product was recrystallized fro $m$ ethanol to afford the desired compound.

\subsection{1. (4a). 3-A mino-5-(4-Bro mobenzylidene)-2-(Pyridin-3- yl)-3,5-Dihydro-4h-Imidazol-4-One}

(yield $53 \%$ \%), (m.p, C $\left.\mathrm{C}^{\circ}, 230-233\right), \mathrm{IR} .(\mathrm{KBr})\left(v, \mathrm{Cm}^{-1}\right)$ 3424-3227 (NH2), 3065 (C-H ar), 3190 (C-H olifen), 1622 (imidazole $\mathrm{C}=\mathrm{O}), 1600-1511(\mathrm{C}=\mathrm{C}$ Ar), $1232(\mathrm{C}-\mathrm{N}) 855$ (para substitution); ${ }^{1} \mathrm{HNMR}$ (DMSO-d6) $\varsigma(\mathrm{ppm}) 8.87$ ( $\mathrm{s}$, $\mathrm{C}=\mathrm{CH}-$ ), 8.43 (s, NH2), $6.91-8.1$ (m, Aro matic protons)

\subsection{2. (4b ). 3-A mino-5-(4-Nitrobenzylidene)-2-(Pyridin-3- yl)-3,5-Dihydro-4h-Imidazol-4-One}

(yield $46 \%)$, (m.p, $\left.\mathrm{C}^{\circ}, 200-201\right)$, IR. ( $\left.\mathrm{KBr}\right)\left(v, \mathrm{Cm}^{-1}\right)$ 3371-3284 (NH2), 3099 (C-H ar), 3222 (C-H olifen), 1626 (imidazole $\mathrm{C}=\mathrm{O}), 1606-1516(\mathrm{C}=\mathrm{C}$ Ar), $1213(\mathrm{C}-\mathrm{N}) 848$ (para substitution); ${ }^{1} \mathrm{HNMR}$ (DMSO-d6) $\varsigma$ (ppm) 8.75 ( $\mathrm{s}, \mathrm{C}=\mathrm{CH}-$ ), 8.51 (s, NH2), $721-8.00$ (m, Aromatic protons)

\subsection{Synthesis of 5-(Arylidene)-3-[(Arylidene) Amino]-2 -(Pyri din-3 -yl)-3,5-Dihydr 0-4H-Imi dazol-4 -One (5a-D)}

The corresponding aryl aldehyde $(0.01 \mathrm{~mol})$ was added to a stirred solution of co mpound $(4 \mathrm{a}-\mathrm{b})(0.01 \mathrm{~mol})$ in absolute ethanol $(20 \mathrm{ml})$ and the mixture was refluxed for $2 \mathrm{~h}$. .After cooling, the mixture was filtered and the solid recrystallized from ethanol to afford the desired compound.

2.6.1. (5a). 5-(4-Bro mobenzylidene)-3-[(4-Nitrobenzy lidene) Amino]-2-(Pyridin-3-y 1)-3,5-Dihydro-4h-Imidazo 1-4One

(yield $83 \%$ ), (m.p, $\left.\mathrm{C}^{\circ}, 242-245\right)$, IR. (KBr) $\left(v, \mathrm{Cm}^{-1}\right) 3028$
(C-H Ar), 3284 (C-H olifen), 1628 (imidazole $\mathrm{C}=\mathrm{O}$ ), 1654 $(\mathrm{C}=\mathrm{N}$, Schiff `s base ) $1604-1519(\mathrm{C}=\mathrm{C}$ Ar), $1267(\mathrm{C}-\mathrm{N}) 850$ (para substitution); ${ }^{1} \mathrm{HNMR}$ (DMSO-d6) $\varsigma(\mathrm{ppm}) 8.65(\mathrm{~s}$, $\mathrm{C}=\mathrm{CH}-), 8.91(\mathrm{~s}, \mathrm{CH}=\mathrm{N}), 6.54-8.12(\mathrm{~m}$, Aromatic protons).

2.6.2. (5b). 5-(4-Bro mobenzlidene)-3[(4-Bro mobenzy lidene) A mino]-2-(Py rid in-3-yl)-3, 5-Dihydro-4H-Imidazol-4-One

(yield $87 \%$ ), (m.p, $\left.\mathrm{C}^{\circ}, 192-194\right), \mathrm{IR} .(\mathrm{KBr})\left(v, \mathrm{Cm}^{-1}\right) 3062$ (C-H Ar), 3190 (C-H olifen), 1631 (imidazole $\mathrm{C}=\mathrm{O}$ ), 1663 $(\mathrm{C}=\mathrm{N}$, Schiff 's base $) 1600-1523(\mathrm{C}=\mathrm{C}$ Ar $), 1278(\mathrm{C}-\mathrm{N}) 841$ (para substitution); ${ }^{1} \mathrm{HNMR}$ (DMSO-d6) $\varsigma$ (ppm) $8.71(\mathrm{~s}$, $\mathrm{C}=\mathrm{CH}-), 8.95(\mathrm{~s}, \mathrm{CH}=\mathrm{N}), 6.43-8.22(\mathrm{~m}$, Aromatic protons).

2.6.3. (5c). 5-(4-Nitrobenzylidene)-3-[(4-Bro mobenzy lidene) Amino]-2-(Pyridin-3-yl)-3,5-Dihydro-4H-Imidazo 1-4 -One

(yield $74 \%$ ), (m.p, $\left.\mathrm{C}^{\circ}, 143-146\right)$, IR. (KBr) $\left(v, \mathrm{Cm}^{-1}\right) 3080$ (C-H Ar), 3122 (C-H olifen), 1632 (imidazole $\mathrm{C}=\mathrm{O}$ ), 1645 $(\mathrm{C}=\mathrm{N}$, Schiff `s base $) 1611-1500(\mathrm{C}=\mathrm{C}$ Ar), $1230(\mathrm{C}-\mathrm{N}) 813$ (para substitution); ${ }^{1} \mathrm{HNMR}$ (DMSO-d6) $\varsigma(\mathrm{ppm}) 8.72(\mathrm{~s}$, $\mathrm{C}=\mathrm{CH}-), 8.98(\mathrm{~s}, \mathrm{CH}=\mathrm{N}), 6.32-7.98(\mathrm{~m}$, Aromatic protons).

2.6.4. (5d). 5-(4-Nitrobenzy lidene)-3-[4-Nit robenzylidene) Amino]-2-(Pyridin-3-y l)-3,5-Dihydro-4H-Imidazo 1-4 -One

(yield 79\%), (m.p, $\left.\mathrm{C}^{\circ}, 145-147\right)$, IR. (KBr) $\left(v, \mathrm{Cm}^{-1}\right) 3069$ (C-H Ar), 3200 (C-H olifen), 1636 (imidazole $\mathrm{C}=\mathrm{O}$ ), 1666 $(\mathrm{C}=\mathrm{N}$, Schiff `s base $) 1601-1514(\mathrm{C}=\mathrm{C}$ Ar $), 1228(\mathrm{C}-\mathrm{N}) 858$ (para substitution); ${ }^{1} \mathrm{HNMR}$ (DMSO-d6) $\varsigma$ (ppm) 8.45 ( s, $\mathrm{C}=\mathrm{CH}-), 8.87(\mathrm{~s}, \mathrm{CH}=\mathrm{N}), 6.51-8.17(\mathrm{~m}$, Aromatic protons).

\subsection{Synthesis of $N-[$ Chlor o (Aryl) \\ Methyl]- $N$-[Aryli dene-5-Ox0-2-(Pyridin-3-yl )-4, 5-Dihydro-1 $H$-Imidazol-1-yl]Benzami de (6a - b )}

To a stirring solution of compound $(5 \mathrm{a}-\mathrm{b})(0.003 \mathrm{~mol})$ in dry benzene $(15 \mathrm{ml})$, benzoyl chloride $(0.003 \mathrm{~mole}, 0.35 \mathrm{~g})$ in benzene $(10 \mathrm{ml})$ was added drop wise, then the mixture was refluxed for $(4 \mathrm{hrs}$.) with stirring. After cooling, the precipitate crystals was filtered and recrystallized from ethanol

\subsection{1. (6a). $N$-[ch loro (4-Bromophenyl) Methyl]- $N$-[4-Nitrobenzy lidene-5-o xo-2-(Py rid in-3-yl )-4, 5-Dihydro-1 $H$-Imidazol-1-yl]Benzamide}

(yield $62 \%),\left(\mathrm{m} . \mathrm{p}, \mathrm{C}^{\circ}\right)(170-173), \mathrm{IR} .(\mathrm{KBr})\left(v, \mathrm{Cm}^{-1}\right)$ 3036 ( $\mathrm{C}-\mathrm{H}$ Ar), 3123 (C-H olifen), 1642 (imidazole $\mathrm{C}=\mathrm{O}), 1651(\mathrm{C}=\mathrm{O}$, amide $) 1588-1481(\mathrm{C}=\mathrm{C}$ Ar $), 1205(\mathrm{C}-\mathrm{N})$ 858 (para substitution); ${ }^{1} \mathrm{HNMR}$ (DMSO-d 6) $\varsigma(\mathrm{ppm}) 8.52(\mathrm{~s}$, $\mathrm{C}=\mathrm{CH}-$ ), 4.76 (s, N-CH-Cl), $6.79-8.42$ (m, Aromatic protons). 


\subsection{2. (6b). $N$-[chloro (4-Nitrophenyl)}

Methyl]- $N$-[4-Bro mobenzylidene-5-o xo-2-(Pyridin-3yl )-4,5-Dihydro-1 $H$-Imidazo 1-1-y l]Ben zamide

(yield $71 \%),\left(\mathrm{m} . \mathrm{p}, \mathrm{C}^{\circ}\right)(190-192), \mathrm{IR} .(\mathrm{KBr})\left(v, \mathrm{Cm}^{-1}\right)$ 3044 (C-H Ar), 3149 (C-H olifen.), 1634 (imidazole $\mathrm{C}=\mathrm{O}), 1664(\mathrm{C}=\mathrm{O}$, amide $) 1603-1524(\mathrm{C}=\mathrm{C}$ Ar $), 1267(\mathrm{C}-\mathrm{N})$ 879 (para substitution); ${ }^{1} \mathrm{HNMR}$ (DMSO-d6) 己 (ppm) 8.75 ( s, C=CH- ), $5.21(\mathrm{~s}, \mathrm{~N}-\mathrm{CH}-\mathrm{Cl}), 715-8.26(\mathrm{~m}$, Aromat ic protons).

\subsection{Synthes is of Aryl $\{[4$-Aryli dene-5-oxo-2-(Pyri din-3- yl)-4,5-Dih ydr o-1 $H$-Imi dazol-1-yl](Benzoyl)Amino\} Methyl Car bami mi dothioate $(7 \mathbf{a}-\mathbf{b})$}

A mixture of compounds $(6 \mathrm{a}-\mathrm{b})(0.005$ mole $)$ thiourea $(0.005 \mathrm{~mole}, 0.44 \mathrm{gm})$ and anhydrous sodium carbonate $(0.005 \mathrm{~mole})$ in absolute ethanol $(20 \mathrm{ml})$ was refluxed for 5 hrs. with stirring and the precipitated crystals was filtered and recrystallized from appropriate solvent.

\subsection{1. (7a). 4-Bro mophenyl\{[4-(4-Nitrobenzylidene-5-o xo- 2-(Pyridin-3-yl)-4,5-Dihydro-1 $H$-Imidazo l-1-yl] (Ben zoyl)A mino Methyl Carbamimidothioate}

(yield $58 \%$ \%), (m.p, $\left.\mathrm{C}^{\circ}\right)(134-136)$, IR. $(\mathrm{KBr})\left(v, \mathrm{Cm}^{-1}\right)$ 3385-3277 (NH$), 3182(\mathrm{NH}) 3049$ (C-H Ar), $3112(\mathrm{C}-\mathrm{H}$ olefene.), 1627 (imidazo le $\mathrm{C}=\mathrm{O}), 1666(\mathrm{C}=\mathrm{O}$, a mide $) 1608$ $1527\left(\mathrm{C}=\mathrm{C}\right.$ Ar), $1297(\mathrm{C}-\mathrm{N}) 858$ (para substitution); ${ }^{1} \mathrm{HNMR}$ (DMSO-d6) 2 (ppm) 8.65 (s, $\mathrm{NH}_{2}$ ), 8.43 ( s, C=CH- ), 4.21 (s, N-CH-S), $6.11-7.86$ (m, Aro matic proton).

2.8.2. (7b ). 4-Nitrophenyl-\{[4-(4-Bro mobenzylidene-5-o xo2-(Pyridin-3-y 1)-4,5-Dihydro-1 H-Imidazo 1-1-y l] (Ben zoyl)A mino Methyl Carbamimidothioate

(yield $65 \%$ ), (m.p, C, $165-167), I R .(\mathrm{KBr})\left(v, \mathrm{Cm}^{-1}\right)$ 3362-3269 ( $\left.\mathrm{NH}_{2}\right), 3169(\mathrm{NH}) 3060$ (C-H Ar), $3102(\mathrm{C}-\mathrm{H}$ olefene.), 1628 (imidazo le $\mathrm{C}=\mathrm{O}), 1643(\mathrm{C}=\mathrm{O}$, amide )1589$1473\left(\mathrm{C}=\mathrm{C}\right.$ Ar), $1294(\mathrm{C}-\mathrm{N}) 823$ (para substitution); ${ }^{1} \mathrm{HNMR}$ (DMSO-d 6) $\varsigma(\mathrm{ppm}) 8.75\left(\mathrm{~s}, \mathrm{NH}_{2}\right), 8.52(\mathrm{~s}, \mathrm{C}=\mathrm{CH}-), 3.86(\mathrm{~s}$, $\mathrm{N}-\mathrm{CH}-\mathrm{S}), 6.46-8.12$ (m, A ro matic proton)).

\subsection{Synthesis of}

$N$-\{1-[(4,6-Diox 0-1, 4,5,6-Tetr ah ydr opyri mi din-2-yl)

Sulfan yl]Benzyl $\}-N$-[Ar yli dene-5-oxo-2-(Pyri di n-3-y 1)-4,5-Dih ydr o-1 $H$-Imi daz ol-1-yl-Benzami de (8 a - b)

A mixture of compounds (7a - b) (0.005mole) diethylmalonate $(0.005 \mathrm{~mole}, 0.8 \mathrm{gm}$.) and anhydrous sodium carbonate $(0.005 \mathrm{~mole})$ in dry benzene $(20 \mathrm{ml})$ was refluxed for $7 \mathrm{hrs}$. with stirring, the product was collected as oily, it is purified by column chromatography with silica gel and a mixture of $\mathrm{EtOH}$ : benzene as eluent.

2.9.1. (8a). $N$-\{1-[(4,6-Dio xo-1,4,5,6-Tetrahydropyrimid in2-yl) Sulfanyl]Benzy 1$\}-N$-[4-Bro mobenzylidene-5oxo-2-(Py rid in-3-yl)-4,5-Dihydro-1H-Imida zol-1-ylBenzamide

(yield 55 \%), (m.p, C $\left.\mathrm{C}^{\circ}, 297-299\right), \mathrm{IR} .(\mathrm{KBr})\left(v, \mathrm{Cm}^{-1}\right) 3248$
(NH) 3044 (C-H Ar), 3176 (C-H olefene.), 2978-2845 (C-H Aliph.), 1701 (Pyrimidine $\mathrm{C}=\mathrm{O}$ ), 1639 (imidazole $\mathrm{C}=\mathrm{O}$ ), $1665(\mathrm{C}=\mathrm{O}$, a mide ) 1600-1501 ( $\mathrm{C}=\mathrm{C}$ Ar), $1241(\mathrm{C}-\mathrm{N}) 825$ (para substitution); ${ }^{1} \mathrm{HNMR}$ (DMSO-d6) $\varsigma$ (ppm) 8.57 (s, $\mathrm{NH}), \quad 8.13(\mathrm{~s}, \quad \mathrm{C}=\mathrm{CH}), \quad 3.56 \quad(\mathrm{~s}, \quad \mathrm{~N}-\mathrm{CH}-\mathrm{S}), \quad 4.42$ (s,NH-CH2-NH) $6.11-7.94$ (m, Aro matic proton).

\subsection{2. (8b ). $N$-\{1-[(4,6-Dio xo-1,4,5,6-Tetrahydropyrimidin- 2-yl) Sulfanyl]Benzy $\}-N$-[4-Nitrobenzylidene-5-o xo- 2-(Pyridin-3-yl)-4,5-Dihydro-1 $H$-Imidazo l-1-y l- Benzamide}

(yield $63 \%$ ), (m.p, $\left.\mathrm{C}^{\circ}, 231-233\right), \mathrm{IR} .(\mathrm{KBr})\left(v, \mathrm{Cm}^{-1}\right) 3202$ (NH) 384 (C-H Ar), 3154 (C-H olefene.), 2990-2849 (C-H Aliph.), 1704 (Pyrimidine $\mathrm{C}=\mathrm{O}$ ), 1631 (imidazole $\mathrm{C}=\mathrm{O}$ ), 1665 ( C=O, a mide ) 1608-1511 (C=C Ar), $1291(\mathrm{C}-\mathrm{N}) 832$ (para substitution); ${ }^{1} \mathrm{HNMR}$ (DMSO-d6) $\varsigma(\mathrm{ppm}) 8.69$ (s, $\mathrm{NH}), \quad 8.21 \quad(\mathrm{~s}, \quad \mathrm{C}=\mathrm{CH}), \quad 3.71 \quad(\mathrm{~s}, \quad \mathrm{~N}-\mathrm{CH}-\mathrm{S}), \quad 4.40$ (s, NH- $\left.\mathrm{CH}_{2}-\mathrm{NH}\right), 6.15-8.02$ (m, Aro matic proton).

\subsection{Synthesis of Ethyl}

$\{[(4 Z)-4-($ Ar ylide ne)-5-ox0-2-(Pyri din-3-yl)-4,5Dihydro-1 H-Imi dazol-1-yl] Amino $\}$ Ace tate 9a-c

The corresponding compound 7 ( 0.01 mole) was refluxed with an equivalent a mount of sodium in absolute ethanol for $2 \mathrm{~h}$. Then, ethyl bromoacetate $(1.81 \mathrm{~g}, 0.01 \mathrm{~mole})$ was added and reflu xed for an additional $5 \mathrm{~h}$. Hav ing evaporated it under reduced pressure, a solid appeared this was recrystallized from ethanol to afford the desired compound.

2.10.1. (9a). ethyl \{[(4Z)-4-(4-bro mobenzylidene)-5-o xo-2(pyridin-3-yl)-4,5-dihydro- $1 H$-imidazol-1-yl]amino acetate

(yield $76 \%$ ), (m.p, $\left.\mathrm{C}^{\circ}, 222-224\right)$, IR. $(\mathrm{KBr})\left(v, \mathrm{Cm}^{-1}\right) 3163$ (NH), 3080 (C-Har), 2993-2885 (C-H alph.), 1741 (ester $\mathrm{C}=\mathrm{O}$ ), 1692 (imidazole $\mathrm{C}=\mathrm{O}), 1265 \quad(\mathrm{C}-\mathrm{O}) ;{ }^{1} \mathrm{HNMR}$ (DMSO-d6) $\varsigma$ (ppm) 1.47-176 (t, CH3-CH2-), 2.25-2.69 (t, $\mathrm{N}-\mathrm{CH} 2), 3.22(\mathrm{~s}, \mathrm{C}=\mathrm{CH}), 3.11-3.29(\mathrm{q}, \mathrm{CH} 2-\mathrm{CH} 3)$, 3.72-3.89, 5.25 (s, N-CH2-CO), 6.42-7.12 (d, 2H, ArH), 7.41-7.92 (d, 2H, ArH), 8.53 (s, 1H, imidazole), 10.62 (s, $\mathrm{NH})$.

2.10.2. (9b). ethyl $\{[(4 Z)-4-(4-n i t r o b e n z y ~ l i d e n e)-5-o x o-2-$ (pyridin-3-yl)-4,5-dihydro- $1 H$-imidazol-1-yl]amino acetate

(yield $85 \%),\left(\mathrm{m} . \mathrm{p}, \mathrm{C}^{\circ}\right)(176-178), \mathrm{IR} .(\mathrm{KBr})\left(v, \mathrm{Cm}^{-1}\right) 3154$ (NH), 3066 (C-Har), 2946-2858 (C-H alph.), 1723 (ester $\mathrm{C}=\mathrm{O}), 1683$ (imidazole $\mathrm{C}=\mathrm{O}), 1612(\mathrm{C}=\mathrm{C}$ alkene) 1290 $(\mathrm{C}-\mathrm{O}) ;{ }^{1} \mathrm{HNMR} \quad$ (DMSO-d6) $\varsigma \quad(\mathrm{ppm}) \quad 1.12-156 \quad(\mathrm{t}$, CH3-CH2-), 2.26-2.35 (t, $\mathrm{N}-\mathrm{CH} 2), 3.18 \quad(\mathrm{~s}, \mathrm{C}=\mathrm{CH})$, 3.41-3.69 (q, CH2-CH3), 5.14 (s, N-CH2-CO), 6.69-7.07 (d, $2 \mathrm{H}, \mathrm{ArH}), 7.47-7.81(\mathrm{~d}, 2 \mathrm{H}$,

\subsection{Synthesis of $N^{\prime}-[($ amino-s ulfanylidyne)} meth yl]-2-\{[(4Z)-4-(aryli dene)-5-ox 0-2 -(pyri din-3-y 1)-4,5-dih ydr o-1 $H$-imi dazol-1-yl] amin o\} acetohy drazi de 10a-b 
A mixture of compound $9 \mathrm{a}-\mathrm{b}(0.01$, mole $)$ and thiosemicarbazide, $(0.91 \mathrm{~g}, 0.01 \mathrm{~mole})$ in ethanol $(25 \mathrm{ml})$ was reflu xed for $8 \mathrm{~h}$. The solution on cooling a solid appeared. This was recrystallized from ethanol to afford the desired compound.

2.11.1. (10a). $N^{\prime}$-[(a mino-sulfanylidyne)methyl] $-2-\{[(4 Z)-4-$ (4-bro mo benzylidene)-5-o xo-2-(pyridin-3-yl)-4,

5-dihydro- $1 H$-imidazol-1-yl] a mino $\}$ acetohydrazide

(yield $61 \%),\left(m . p, \mathrm{C}^{\circ}\right)(212-214)$, IR. $(\mathrm{KBr})\left(\mathrm{v}, \mathrm{Cm}^{-1}\right)$ 3410-3375 (NH2), 3247 (thiosemicarbazide $\mathrm{NH}$ ), $3199(\mathrm{NH})$, 3056 (C-Har), 2971-2850 (C-H alph.), 1688 (imidazole $\mathrm{C}=\mathrm{O}$ ), 1641 (a mide $\mathrm{C}=\mathrm{O}), 1243(\mathrm{C}=\mathrm{S}) ;{ }^{1} \mathrm{HNMR}$ (DMSO-d6) $\varsigma(\mathrm{ppm}) 2.16-2.52(\mathrm{t}, \mathrm{N}-\mathrm{CH} 2), 3.18(\mathrm{~s}, \mathrm{C}=\mathrm{CH}), 4.83(\mathrm{~s}$, $\mathrm{N}-\mathrm{CH} 2-\mathrm{CO}), 6.55$ (s, NH2), 6.78-6.91 (d, 2H, ArH), 7.34-7.85 (d, 2H, ArH), 8.86 (s, 1H, imidazole), 10.15 (s, $\mathrm{NH}), 10.84$ (s, N-NH-CS), 11.42 (s, CO-NH-N).

2.11.2. (10b). $N^{\prime}$-[(a mino-sulfanylidyne)methyl]-2$\{[(4 Z)-4-(4-n i t r o$ ben zylidene)-5-o xo-2-(pyrid in-3yl)-4,5-d ihydro- $1 H$-imidazo l-1-yl] a mino acetohydrazide

(yield $59 \%),\left(\right.$ m.p, $\left.\mathrm{C}^{\circ}, 254-256\right)$, IR. $(\mathrm{KBr})\left(v, \mathrm{Cm}^{-1}\right)$ 3421-3397 (NH2), 3286 (thiosemicarbazide $\mathrm{NH}$ ), $3226(\mathrm{NH})$, 3060 (C-Har), 2978-2899 (C-H alph.), 1704 (imidazole $\mathrm{C}=\mathrm{O}$ ), 1664 (amide $\mathrm{C}=\mathrm{O}), 1243(\mathrm{C}=\mathrm{S}) ;{ }^{1} \mathrm{HNMR}$ (DMSO-d6) $\varsigma(\mathrm{ppm})$ 2.32-2.77 (t, N-CH2), $3.21(\mathrm{~s}, \mathrm{C}=\mathrm{CH}), 4.45$ (s, $\mathrm{N}-\mathrm{CH} 2-\mathrm{CO}), \quad 5.93$ (s, NH2), 6.51-6.78 (d, 2H, $\operatorname{ArH})$, 7.43-7.92 (d, 2H, ArH), 8.43 (s, 1H, imidazole), 10.28 (s, NH), 10.67 (s, N-NH-CS), 11.19 (s, CO-NH-N).

\subsection{Synthesis of $(5 Z)-3-\{[(5-a m i n o-1,3,4-$ thiadi azol-2- yl)methyl $]$ amino\}-5-(arylide ne)-2-(pyri din-3-yl)-3, 5-dihydr o-4H-i mi dazol-4-one 11 a-b}

Corresponding compounds $10 \mathrm{a}-\mathrm{b}(0.01 \mathrm{~mole})$ was dissolved in cold conc. Sulfuric acid $(10 \mathrm{ml})$ and stirred at room temperature for $24 \mathrm{~h}$. Then, reaction mixture was poured into crushed ice and diluted with water, the precipitate was filtered, washed with water and recrystallized from ethanol to afford the desired compound.

\subsection{1. (11a 0. (5Z)-3-\{[(5-a mino-1,3,4-thiad iazol-2-yl)} methyl]a mino -5-(4-bro mobenzy lidene)-2-(pyridin3-yl)-3,5-d ihydro-4H-imidazo l-4-one

(yield $63 \%$ ), (m.p, $\left.\mathrm{C}^{\circ}, 174-176\right)$, IR. $(\mathrm{KBr})\left(\mathrm{v}, \mathrm{Cm}^{-1}\right)$ 3432-3386 (NH2), 3210 (NH), 3042 (C-Har), 2986-2879 (C-H alph.), 1651 (imidazole $\mathrm{C}=\mathrm{O}$ ); ${ }^{1} \mathrm{HNMR}$ (DMSO-d 6) $\varsigma$ (ppm) : 3.62 (s, $\mathrm{C}=\mathrm{CH}), 4.85$ (s, N-CH2-Th iadiazole), 6.11 (s, NH2), 6.51-6.82 (d, 2H, ArH), 7.45-7.97 (d, 2H, ArH), 8.23(s, 1H,imidazole), 10.31 (s, NH).

\subsection{2. (11b). (5Z)-3-\{[(5-a mino-1,3,4-thiad iazol-2-yl)} methyl]a mino -5-(4-bromobenzy lidene)-2-(pyridin3-yl)-3,5-d ihydro-4H-imidazo l-4-one

(yield $71 \%),\left(m . p, C^{\circ}, 281-283\right), \quad I R . \quad(\mathrm{KBr})\left(v, \mathrm{Cm}^{-1}\right)$ 3427-3388 (NH2), 3215 (NH), 3049 (C-Har), 2991-2890
(C-H alph.), 1701 (imidazole $\mathrm{C}=\mathrm{O}$ ); ${ }^{1} \mathrm{HNMR}$ (DMSO-d 6) $\varsigma$ (ppm) : $3.29(\mathrm{~s}, \mathrm{C}=\mathrm{CH}), 4.58$ (s, N-CH2-Thiadiazole), 6.21 (s, NH2), 6.76-6.81 (d, 2H, ArH), 7.46-7.78 (d, 2H, ArH), 8.25(s, $1 \mathrm{H}$,imidazole), 10.66 (s, NH).

\subsection{Synthesis of 2-\{[(4Z)-4-(aryli dene)-5-0xo-2 - (pyridin-3-yl)-4,5-dihydr o- $1 H$-imi dazol-1-yl] amino\} acetohydr azi de 12a-b}

A mixture of compound $9 \mathrm{a}-\mathrm{b}(0.01$, mole $)$ and hydrazine hydrate $(99 \%, 0.32 \mathrm{~g}, 0.01 \mathrm{~mole})$ in ethanol $(25 \mathrm{ml})$ was refluxed for $8 \mathrm{~h}$. The solution on cooling a solid appeared. This was recrystallized from ethanol to afford the desired compound.

2.13.1. (12a). 2-\{[(4Z)-4-(4-bromobenzylidene)-5-oxo-2(pyridin-3-yl)-4,5-dihydro- $1 H$-imidazol-1-yl]a mino acetohydrazide

(yield $74 \%),\left(m . p, C^{\circ}, 162-164\right), \quad I R . \quad(\mathrm{KBr})\left(v, \mathrm{Cm}^{-1}\right)$ 3400-3374 (NH2), 3230 (NH), 3071 (C-Har), 2948-2826 (C-H alph.), 1699 (imidazo le $\mathrm{C}=\mathrm{O}$ ), 1670 (a mide $\mathrm{C}=\mathrm{O}$ ), 1232 (C-N); ${ }^{1} \mathrm{HNMR}$ (DMSO-d6) $\varsigma$ (ppm) 2.36-2.67 (t, N-CH2), $3.43(\mathrm{~s}, \mathrm{C}=\mathrm{CH}), 4.28$ (s, N-CH2-CO), 6.22 (s, NH2), 6.71-7.18 (d, 2H, ArH), 7.42-7.893 (d, 2H, A rH), $8.52(\mathrm{~s}, 1 \mathrm{H}$, imidazo le), 10.74 (s, NH), 11.91 (s, CO-NH-N).

\subsection{2. (12b). 2-\{[(4Z)-4-(4-nitrobenzylidene)-5-o xo-2-} (pyridin-3-yl)-4,5-dihydro- $1 H$-imidazol-1-yl]a mino acetohydrazide

(yield $62 \%), \quad\left(m . p, C^{\circ}, 186-188\right), \quad I R . \quad(\mathrm{KBr})\left(v, \mathrm{Cm}^{-1}\right)$ 3431-3383 (NH2), 3210 (NH), 3088 (C-Har), 2992-2879 (C-H alph.), 1690 (imidazo le $\mathrm{C}=\mathrm{O}$ ), 1657 (a mide $\mathrm{C}=\mathrm{O}$ ), 1242 (C-N); ${ }^{1} \mathrm{HNMR}$ (DMSO-d6) $\varsigma$ (ppm) 2.56-2.89 (t, N-CH2), 3.40(s, $\mathrm{C}=\mathrm{CH}), 4.58$ (s, N-CH2-CO), 6.52 (s, NH2), 6.70-6.82 (d, 2H, ArH), 7.44-7.89 (d, 2H, ArH), 8.47 (s, 1H, imidazo le), 10.57 (s, NH), 11.02 (s, CO-NH-N).

\subsection{Synthesis of (5Z)-5-(aryli dene)-2-(pyridin-3-yl)-3- $\{[(5-$ sulfan yl-1,3,4-oxadi azol-2-yl) methyl] amino $\}-3$, 5-dihy dr 0-4H-i mi dazol-4-one 13a-b}

Corresponding compound $12 \mathrm{a}-\mathrm{b}(0.01 \mathrm{~mole})$ and $\mathrm{CS}_{2}(0.6$ $\mathrm{ml}, 0.01 \mathrm{~mole})$ were added to a solution of $\mathrm{KOH}(0.56 \mathrm{~g}, 0.01$ mole) in $(30 \mathrm{ml})$ ethanol. The reaction mixture was refluxed for $3 \mathrm{~h}$. After evaporation it in reduced pressure to dryness, a solid obtained. This was dissolved in $200 \mathrm{ml} \mathrm{H}_{2} \mathrm{O}$ and acidified with conc. $\mathrm{HCl}$. The precipitate was filtered off, washed with water and recrystallized from ethanol to afford the desired compound.

2.14.1. (13a0. (5Z)-5-(4-bro mobenzy lidene)-2-(pyrid in-3yl)-3-\{[(5-sulfanyl-1,3,4-o xadiazol-2-yl)methyl] amino \}-3,5-d ihydro-4H-imidazo 1-4-one

(yield $44 \%$ ), (m.p, C $\left.\mathrm{C}^{\circ}, 193-195\right)$, IR. (KBr) $\left(v, \mathrm{Cm}^{-1}\right) 3198$ (NH), 3075 (C-Har), 2982-2893 (C-H alph.), 2466 (SH), 1685 (imidazo le $\mathrm{C}=\mathrm{O}), 1291(\mathrm{C}=\mathrm{S}) ;{ }^{1} \mathrm{HNMR}$ (DMSO-d6) $\varsigma$ (ppm) 2.41-2.64 (t, N-CH2), $3.34(\mathrm{~s}, \mathrm{C}=\mathrm{CH}), 4.45$ (s, 
N-CH2-o xadiazo le), 6.71-6.94 (d, 2H, ArH), 7.59-7.93 (d, 2H, ArH), 8.74 (s, 1H, imidazo le), 10.61 (s, NH), 12.04 (s, $\mathrm{NH}+\mathrm{SH}$ of oxadiazole).

\subsection{2. (13b). (5Z)-5-(4-nitrobenzylidene)-2-(pyrid in-3-yl)-} 3- $\{[(5$-sulfanyl-1,3,4-o xad ia zol-2-yl)methyl $]$ a mino $\}$ -3,5-dihydro-4H-imidazol-4-one

(yield $60 \%$ ), (m.p, $\left.\mathrm{C}^{\circ}\right)(255-257), \mathrm{IR} .(\mathrm{KBr})\left(v, \mathrm{Cm}^{-1}\right) 3206$ (NH), 3053 (C-Har), 2991-2890 (C-H alph.), 2465 (SH), 1668 (imidazole $\mathrm{C}=\mathrm{O}), 1272(\mathrm{C}=\mathrm{S}) ;{ }^{1} \mathrm{HNMR}$ (DMSO-d6) $\varsigma$ (ppm) 2.33-3.02 (t, N-CH2), $3.61(\mathrm{~s}, \mathrm{C}=\mathrm{CH}), 4.42(\mathrm{~s}$, N-CH2-oxadiazole), 6.54-6.69 (d, 2H, ArH), 7.41-7.87 (d, $2 \mathrm{H}, \mathrm{ArH}), 8.27$ (s, 1H, imidazole), 10.28 (s, NH), 12.94 (s, $\mathrm{NH}+\mathrm{SH}$ of oxadiazole).

\subsection{Synthesis of (5Z)-3-\{[(4-ami no-5-s ulfanyl-4 H-1,2, 4-triazol-3-yl )methyl] amin o\}-5-(aryli dene)-2- (pyri din- 3-yl)-3,5-di hydr o-4H-i mi daz ol-4-one 14a-b}

Corresponding compound $12 \mathrm{a}-\mathrm{b}(0.01 \mathrm{~mole})$ and CS2 $(0.6 \mathrm{ml}, 0.01 \mathrm{~mole})$ in $(20 \mathrm{ml})$ ethanol. The reaction mixture was stirred for $12 \mathrm{~h}$. Then, diethyl ether $(18 \mathrm{ml})$ was added to it. The precipitated solid thus obtained was filtered, washed with cold diethyl ether, without isolation and purification dis solved in water $(10 \mathrm{ml})$, hydrazine hydrate $(99 \%)(0.34 \mathrm{~g}$, 0.01 mole) was added. The reaction mixture was refluxed for 1 h. cooled, diluted with water and acidified with acetic acid. The precipitate was filtered off, washed with water and recrystallized from ethanol to afford the desired compound.

2.15.1. (14a).(5Z)-3-\{[(4-a mino-5-sulfanyl-4H-1,2, 4-triazol -3-yl)methyl]amino $\}-5-(4-$ bromobenzylidene)-2-(pyrid in-3-yl)-3, 5-dihydro- $4 H$-imidazol-4-one

( yield $66 \%),\left(\mathrm{m} . \mathrm{p}, \mathrm{C}^{\circ}, 204-206\right), \mathrm{IR} .(\mathrm{KBr})\left(v, \mathrm{Cm}^{-1}\right)$ 3412-3352 (NH2), 3270 (NH), 3066 (C-Har), 2982-2887 (C-H alph.), 2440 (SH), 1692 (imidazole $\mathrm{C}=\mathrm{O}), 1255(\mathrm{C}=\mathrm{S})$; ${ }^{1} \mathrm{HNMR}$ (DMSO-d 6) $\varsigma$ (ppm) 2.43-2.69 (t, N-CH2), 3.40 (s, $\mathrm{C}=\mathrm{CH}), 4.91$ (s, N-CH2-Triazo le), 6.52 (s, NH2), 6.72-6.98 (d, 2H, ArH), 7.49-7.81 (d, 2H, A rH), 10.69 (s, NH), 13.01 (s, $\mathrm{NH}+\mathrm{SH}$

\subsection{2. (14b). (5Z)-3- $\{[(4-a$ mino-5-sulfanyl-4H-1,2,4- triazol-3-yl)methyl]amino $\}-5-(4-$ mitrobenzylidene)-2-(pyrid in-3-yl)-3, 5-dihydro- $4 H$-imidazol-4-one.}

(yield $57 \%$ \%), (m.p, $\left.\mathrm{C}^{\circ}, 290-292\right)$, IR. $(\mathrm{KBr})\left(v, \mathrm{Cm}^{-1}\right)$ 3439-3312 (NH2), 3178 (NH), 3059 (C-Har), 2985-2870 (C-H alph.), 2427 (SH), 1704 (imidazole $\mathrm{C}=\mathrm{O}), 1219(\mathrm{C}=\mathrm{S})$; ${ }^{1} \mathrm{HNMR}$ (DMSO-d 6) $\varsigma$ (ppm) 2.51-2.78 (t, N-CH2), 3.35 (s, $\mathrm{C}=\mathrm{CH}$ ), 4.62 (s, N-CH2-Triazole), 6.13 (s, NH2), 6.71-6.95 (d, 2H, ArH), 7.51-7.87 (d, 2H, ArH), 8.82 (s, 1H, imidazole), $10.58(\mathrm{~s}, \mathrm{NH}), 13.10(\mathrm{~s}, \mathrm{NH}+\mathrm{SH}$ of triazole $)$.

\section{Results and Discussion}

Schemes (1) were summarized the synthes is of different derivatives of nicotinic acid.

Nicotinoyl chloride (1) was synthesized by treatment of nicotinic acid with thionyl chloride. The reaction is followed by the appearance of $\left(v_{\mathrm{C}=\mathrm{O}}\right)$ absorption bands at (1799) $\mathrm{cm}^{-1}$ in their spectra.[(Pyridyl-3-yl-carbonyl amino]acetic acid (2)[13] have been synthesized by the reaction of compound (1) with glycine in the presence of sodium hydro xide through nucleophilic displacement mechanis $\mathrm{m}\left(\mathrm{SN}_{2}\right)$. The reaction is followed by decreasing of absorption band for $\left(v_{\mathrm{C}=\mathrm{O}}\right)$ at 1741 $\mathrm{cm}^{-1}$ and appearance of new absorption band at $3180 \mathrm{~cm}^{-1}$ due to $\left(v_{\mathrm{NH}}\right)$. In the ${ }^{1} \mathrm{H}$ NMR spectra, the proton signals due to $\left(\mathrm{CO}-\mathrm{CH}_{2}-\mathrm{NH}\right)$ was recorded at $4.42 \mathrm{ppm}$ integrating for two protons. The treatment of compound (2) with aryl aldehyde in presence of acetic acid and acetic anhydride led to the formation of 4-(arylidene)-2-(pyridin-3-yl)-1,3-o xazo 1-5(4H)-one(3a- b).[14] Compounds $(3 \mathrm{a}-\mathrm{b})$ have been identified by IR spectrum which it showed the appearance of characteristic absorption bands near 1755-1770 $\mathrm{cm}^{-1}$ which belonged to the oxazol-5(4H)-one carbonyl group (oxazole, $v_{\mathrm{C}=\mathrm{O}}$ ), and at 3123-3220 $\mathrm{cm}^{-1}$ due to (olifin ic $v_{\mathrm{CH}}$ ), ${ }^{1} \mathrm{H} \mathrm{NMR}$ spectra showed signals at $8.82-8.90 \mathrm{ppm}$ due to $(\mathrm{CH}$, olifenic) and at 6.21-8.10 ppm which belonged to aromatic protons. Refluxing compounds $(3 \mathrm{a}-\mathrm{b})$ with hydrazine hydrate $(99 \%)$ for $20 \mathrm{hrs}$ offered good yields of the corresponding derivatives $(4 a-b)$. The IR spectra of compounds $(4 \mathrm{a}-\mathrm{b})$ displaced peaks at $1622-1626 \mathrm{~cm}^{-1}$, $3227-3424 \mathrm{~cm}^{-1}$ for (imidazole, $v_{\mathrm{C}=\mathrm{O}}$ ) and $\left(\mathrm{v}_{\mathrm{NH} 2}\right)$ functions respectively. ${ }^{1} \mathrm{H}$ NMR spectra showed signals at 8.43-851 ppm due to $\left(\mathrm{NH}_{2}\right)$, at $8.75-8.87 \mathrm{ppm}$ for $(\mathrm{CH}$, olifenic $)$ and at $6.91-8.1 \mathrm{ppm}$ which belonged to aromatic protons. The formation of Schiff's bases $(5 \mathrm{a}-\mathrm{d})$ was confirmed by the absence of absorption bands near $3227-3424 \mathrm{~cm}^{-1}$ for $\left(v_{\mathrm{NH} 2}\right)$ and presence of absorption bands at $1645-1666 \mathrm{~cm}^{-1}$ due to $\left(v_{\mathrm{C}=\mathrm{N}}\right)$ stretching. ${ }^{1} \mathrm{HNMR}$ spectra of compound $(5 \mathrm{a}-\mathrm{d})$ show the new signals observed at 8.87-8.98 ppm integrating for $(\mathrm{CH}=\mathrm{N})$ and at $6.32-8.22 \mathrm{ppm}$ integrating for protons assigned to aryl groups.

When the Schiff's bases $(5 a-b)$ were treated with benzoyl chloride (scheme 2$)$ in boiling benzene, derivative $(6 a-b)$ was obtained in good yield. The IR spectrum ind icated the presence of a $\left(v_{\mathrm{CON}}\right)$ function (1651-1664) $\mathrm{cm}^{-1}$ and at 1642-1634 $\mathrm{cm}^{-1}$ due to(imidazole $\left(v_{\mathrm{C}=\mathrm{O}}\right)$ in addition to (olifinic $v_{\mathrm{CH}}$ ) at 3123-3149 $\mathrm{cm}^{-1} .{ }^{1} \mathrm{H}$ NMR spectra showed signals at $8.52-8.75 \mathrm{ppm}$ due to $(\mathrm{CH}$, olifenic), at 6.79-8.42 ppm which belonged to aro matic protons and at 4.76-5.21 for ( $\mathrm{N}-\mathrm{CH}-\mathrm{Cl})$. On the other hand, when the derivatives $(6 \mathrm{a}-\mathrm{b})$ was treated with thiourea, derivatives $(7 \mathrm{a}-\mathrm{b})$ was obtained in good yield. The IR spectra indicated the presence of a doublet absorption bands near (3385-3269) $\mathrm{c} \mathrm{m}^{-1}$ for $\left(v_{\mathrm{NH} 2}\right)$. . ${ }^{1} \mathrm{H}$ NMR spectra showed signals at $8.65-8.75 \mathrm{ppm}$ due to $(\mathrm{NH} 2)$, at $6.11-8.12 \mathrm{ppm}$ which belonged to aromatic protons and at 3.86-4.21 for ( N-CH-S). 


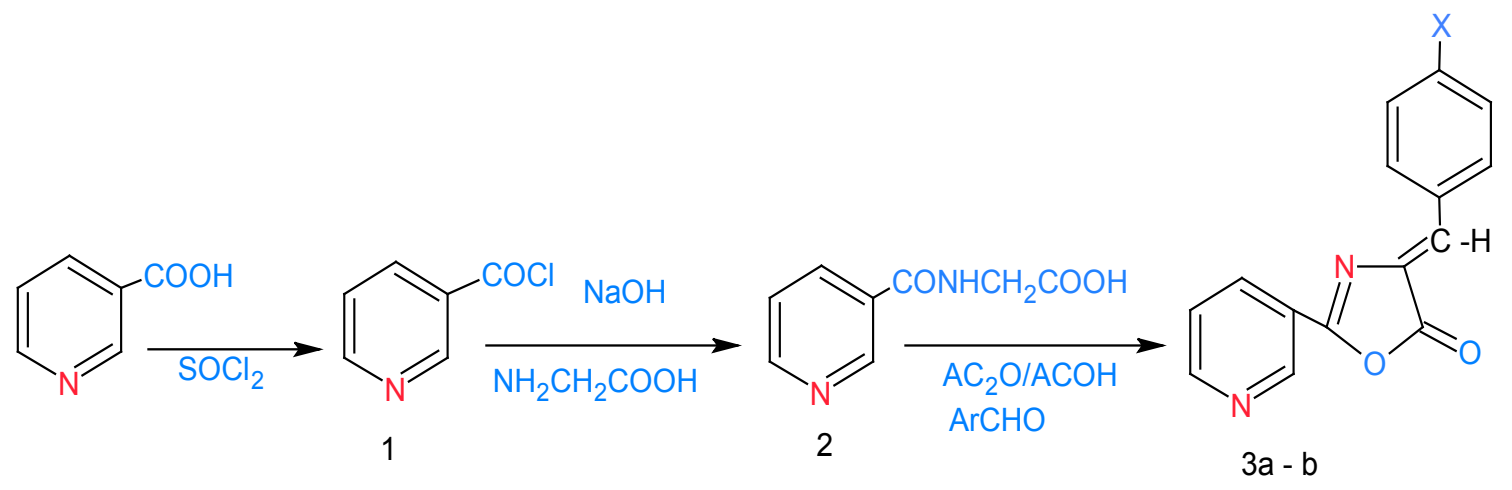
$\mathrm{X}=\mathrm{Br}, \mathrm{NO}_{2}$

Scheme 1. shows synthesized of compounds $3 a-b$<smiles>[X]c1ccc(/C=C2\N=C(c3cccnc3)OC2=O)cc1</smiles>

$3 a-b$<smiles>[X]c1ccc(/C=C2\N=C(c3cccnc3)N(N)C2=O)cc1</smiles>

$4 a-b$

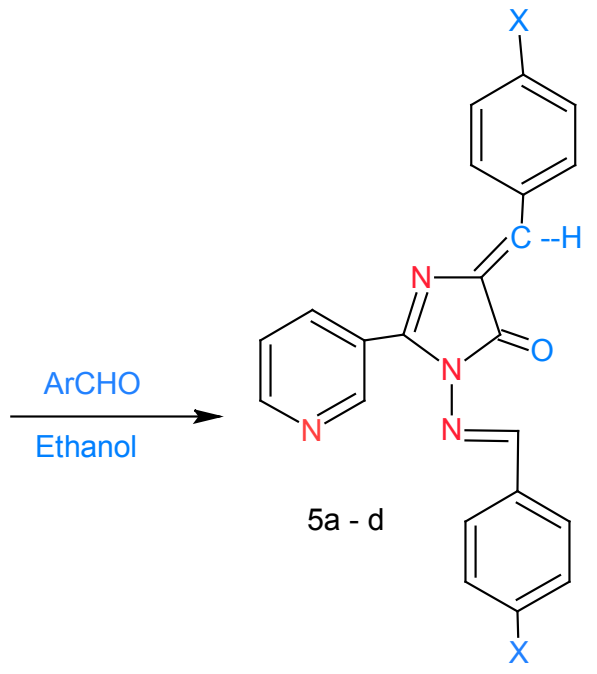

$\mathrm{X}=\mathrm{Br}, \mathrm{NO}_{2}$

Scheme 2. shows synthesized of compounds 5a- b<smiles>[X]c1ccc(/C=N/N2C(=O)/C(=C/c3ccc([X])c(/C=C4\N=C(c5cccnc5)N(N(C(=O)c5ccccc5)C(c5ccc([X])cc5)c5ccc([R19])cc5)C4=O)c3)N=C2c2cccnc2)cc1</smiles><smiles>[X]c1ccc(/C=C2\N=C(c3cccnc3)N(N(Cc3ccccc3)C(C)c3ccc([Z])cc3)C2=O)cc1</smiles>
$\mathrm{X}=\mathrm{Br}, \mathrm{NO}_{2}$

Scheme 3. shows synthesized of compounds $6 a-d$ and $7 a-d$ 


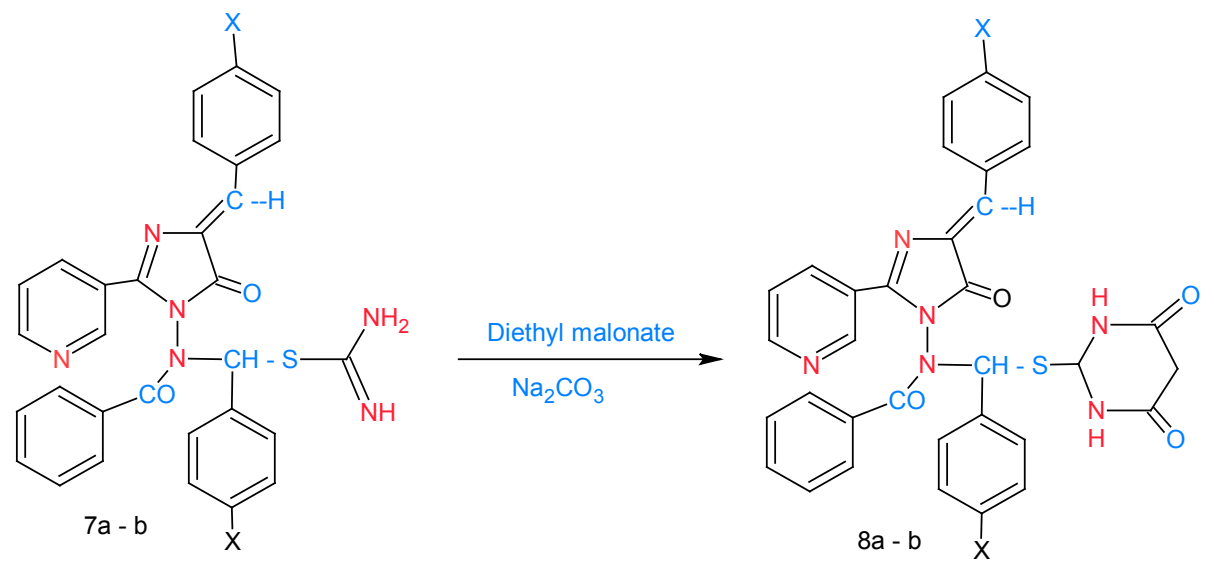

$\mathrm{X}=\mathrm{Br}, \mathrm{NO}_{2}$

Scheme 4. shows synthesized of compounds $8 \mathrm{a}-\mathrm{b}$

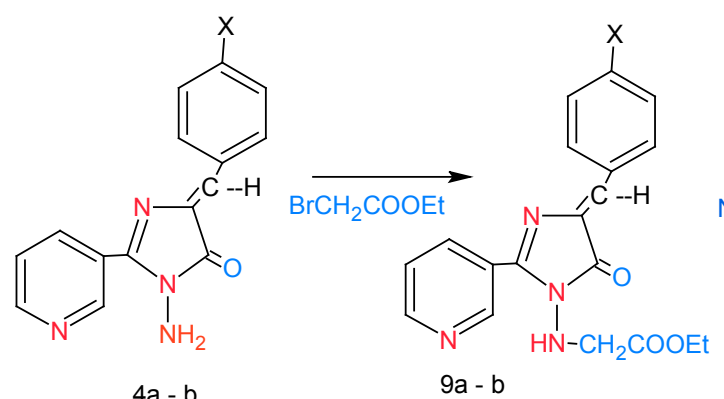

$4 a-b$<smiles>[X]c1ccc(C2N=C(c3cccnc3)N(NCC(N)=O)C2=O)cc1</smiles>

$10 a-b$<smiles></smiles><smiles></smiles>

$13 a-b$

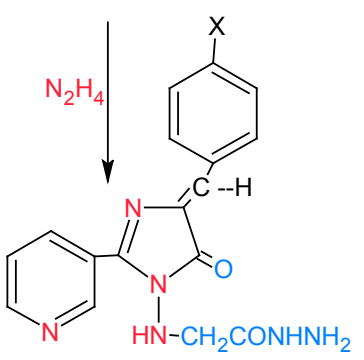

$12 \mathrm{a}-\mathrm{b}$ $11 \mathrm{a}-\mathrm{b}$<smiles>[Y][14CH2]c1nnc(N)s1</smiles>
$\mathrm{KOH}$<smiles>C=C(C)C1N=C(c2cccnc2)N(N)C1=O</smiles>

$14 a-b$

$$
\mathrm{X}=\mathrm{Br}, \mathrm{NO}_{2}
$$

Scheme 5. shows synthesized of compounds $(9 a-b)-(14 a-b)$

Oxidative cyclization of derivative (7a - b) by diethylmalonate, produced the corresponding pyrimidine derivatives $(8 \mathrm{a}-\mathrm{b})$ in good yield, The IR spectra of compounds $(8 \mathrm{a}-\mathrm{b})$ exhibited a $\left(v_{\mathrm{CH}}\right.$ Aliph. $)$ stretching vibration at $2990-2845 \mathrm{~cm}^{-1}$ and $\left(v_{\mathrm{NH}}\right)$ stretching vibration at 3202-3242 $\mathrm{cm}^{-1}$. ${ }^{1} \mathrm{H}$ NMR spectra showed signals at 4.40-4.42 ppm due to (NH-CH2-NH) and at 6.11-8.02 ppm which belonged to aromatic protons.

The formation of compounds $9 a-b$ was confirmed by the presence of a sharp absorption near $1741-1723 \mathrm{~cm}-1$ for the ester $\mathrm{C}=\mathrm{O}$ and at $1265-1290 \mathrm{~cm}-1$ due to $\mathrm{C}-\mathrm{O}$ stretching. In the $1 \mathrm{H}$ NMR spectra, the proton signals due to ethyl group of ester $\mathrm{O}-\mathrm{CH} 2 \mathrm{c}-\mathrm{CH} 3 \mathrm{~d}$ were recorded between 1.12-1.76 ppm, integrating for three protons (d) and 3.11-3.69 ppm 
integrating for two protons (c). The treatment of compounds $9 \mathrm{a}-\mathrm{c}$ with thiosemicarbazide and hydrazine hydrate give compounds 10a-c[15] and compounds 12a-b[15], respectively. The spectral data of compounds $10 \mathrm{a}-\mathrm{b}$ and 12a-b are given in the Experimental section. Acid hydrazides ' are useful intermediates leading to the formation of some heterocyclic ring such as 1,3,4-o xadiazoles 13a-b, and 1,2,4-triazo les $14 a-b$. the treatment of compounds 11a-b with conc. $\mathrm{H}_{2} \mathrm{SO}_{4}$ afforded compounds 11a-b The formation of the 1,2,4-triazole derivatives $11 \mathrm{a}-\mathrm{b}$ as confirmed by the presence of weak absorptions near 2,570-2,630 cm-1 for SH and $1,230-1,300 \mathrm{~cm}-1$ due to $\mathrm{C}=\mathrm{S}$. The structures of compounds 15 a-c were confirmed by ${ }^{1} \mathrm{H}-\mathrm{NMR}$ and IR The data are shown the Experimental section.

\section{REFERENCES}

[1] Maillard J., Vincent M., Morin R., Benard M. " Hypnotic and sedative drug, 2-(O-hydoxyphenyl) -1,3,4-oxadiazole"; French Patent M 379: Chem Abstr: 57, 15251, (1962)

[2] Srinivas A., Raju J. , Rama G., Sarangapani M."Synthesis and in vivo anti-inflammatory activity of a novel series of benzoxazole derivatives" Der Chemica Sinica, 1 (3): 157-168, (2010).

[3] Priy anka L., Nagori B., Nikhil B., Anju G., Stuti G. and Nisha Singh "Benzoxazole: The molecule of diverse biological activities" J. Chem. Pharm. Res., 2011, 3(3):302-311

[4] Kennedy D.A., Summers L. A. "Chemical constitution and activity of Herbicides. Part XIV. Reduction potential and herbicidal activity of 4, 4-(1,3,4-thiadiazole-2,5-diyl)-and 4,4-(1,3,4-oxadiazole-2,5-diyl) bis (1-methylpyridinium) diiodides", J Heterocy cl Chem 409-410, (1981)

[5] Kumari S., Pramod K. Sh. and Nitin K. "Imidazole and its biological activities"; Der Chemica Sinica, 1 (3): 36-47,
(2010).

[6] Saeed-ur-R., Muhammad K., Sadia R., Alia F. and Shahnawaz "Synthesis, characterization and antimicrobial studies of transition metal complexes of imidazole derivatives" Bull. Chem. Soc. Ethiop., 24(2), 201-207, (2010)

[7] Priya V. F., Girish K. S. and Balakrishna K. "Solvent-free microwave-assisted synthesis of oxadiazoles containing imidazole moiety" J. Chem. Sci., Vol. 119, No. 1, , pp.41-46 January (2007)

[8] Eduardo A. Ve'liz and Peter A. Beal "6-Bromopurine Nucleosides as Reagents for Nucleoside Analogue" Synthesis J. Org. Chem., 66, 8592-8598, (2001)

[9] Sahar A. Kadem "Synthesis of New Imidazole Derivative Mustansiriya J. Sci., 3(17), 50-58(2010)

[10] Sahar A. Kadhom, Shaemaa H. Abdul sada, Ghasak J. Kashtal Abdul- Jabber K. Atia and Mohammed A. Synthesis of New heterocyclic compounds derived from "2-amino-2-(4-hydroxy phenyl) acetic acid and study of their effects on Monoamineoxidase (MAO) activity" Al-Mustansiriy a J. Sci., 3(18), 21-31(2010)

[11] Eldon E. Baird and Peter B. "Synthesis of Polyamides Containing Imidazole and Pyrrole Amino Acids" J. Am. Chem. Soc. 118, 6141-6146, (1996)

[12] Tomaž T. and Bojan V.," Synthesis of alkyl 2-(benzoyl amino)-3-(4,5-dicy ano-1H-imidazol-1-yl)propenoate" ARKTVOC (XiV) 96-101, (2005)

[13] Vogel, A. I., hippuric acid " A Text Book of Practical Organic Chemistry ", 4th Ed., P 561, Longmans Green and Co. INC., LONDON, ( 1954).

[14] Abdul Jabbar K. Atia. "Synthesis and Antibacterial Activities of Metronidazole and new Imidazole Derivatives", Molecules, 14, 2431-4446; doi: 10.3390/ molecules 14072431, (2009)

[15] Borisch, K.; Diele, S; Goring, P.; Kresse, H.; Tschierske, C. Synthesis of Novel Organometallic Polymers Based Upon N-Heterocy clic Carbenes. J. Mater. Chem., 8, 529-543.(1998) 